\title{
Nagelmykose
}

\section{Wasserlöslicher Nagellack wirkt auch gegen Sporen}

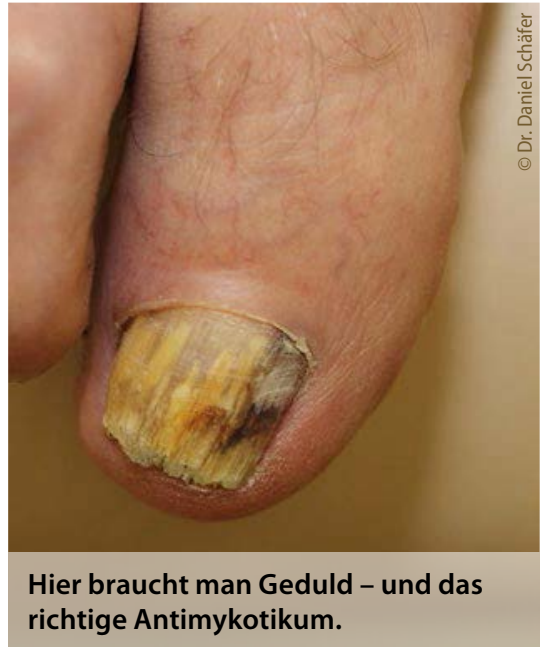

- Nagelpilzerreger wie Trichophyton rubrum haben die unangenehme Eigenschaft, immer wieder zu kommen. Eine
Quelle dieser Rezidive sind die im Nagelbett befindlichen, extrem widerstandsfähigen Pilzsporen. Wie Prof. Hans-Jürgen Tietz vom Institut für Pilzkrankheiten und Mikrobiologie in Berlin betonte, stellen die Sporen ein zentrales Problem bei der Therapie der Nagelmykose dar und müssen unbedingt beseitigt werden, um Rezidive zu verhindern.

Der medizinische Nagellack Ciclopo$\mathrm{li}^{\circledR}$ eignet sich sehr gut zur topischen Therapie von Nagelmykosen. Einerseits bekämpft der bewährte Wirkstoff Ciclopirox sowohl die Pilze als auch deren Sporen. Andererseits ermöglicht die patentierte Lacktechnologie (ONY-FLEX ${ }^{\circledast}$ ) mit dem Biopolymer Hydroxypropylchitosan (HPCH) einen raschen Transport des Wirkstoffs in den Nagel und das $\mathrm{Na-}$ gelbett. Der wasserlösliche Lack wird abends aufgetragen und bildet einen unsichtbaren Film, der am nächsten Morgen einfach abgewaschen wird.

Eine randomisierte Studie verglich Ciclopoli-Nagellack (täglich angewendet) mit einem handelsüblichen 5\% Amorolfin Nagellack auf Acrylatbasis (zweimal wöchentlich) bei 137 Patienten mit Onychomykose. Nach 48 Wochen Behandlung zeigten mit 35,0\% vs. $11,7 \%$ deutlich mehr Patienten der Ciclopoli-Gruppe eine Komplett-Heilung (Konversion zu negativer Mikroskopie, negative Pilzkultur und $100 \%$ geheilter Nagel.

\section{Dr. Marion Hofmann-Aßmus}

- Mykologie-Seminar "Dermatologische Kernkompetenz Nagelerkrankungen und Mykologie", Tagung Dermatologische Praxis; Frankenthal, März 2017 (Veranstalter: Taurus)

\section{Neuer tetravalenter \\ Grippeimpfstoff steht bereit}

Derzeit sind die meisten saisonalen Grippeimpfstoffe trivalent, was bedeutet, dass sie vor drei Subtypen des Influenzavirus schützen: vor zwei A-Stämmen und einer einzigen B-Linie (B/Victoria oder B/Yamagata). Allerdings wurde über die jüngsten Grippesaisons hinweg durchwegs eine Ko-Zirkulation beider B-Linien beobachtet. Sanofi Pasteur gibt bekannt, dass sein neuer tetravalenter Grippeimpfstoff Vaxigrip Tetra ${ }^{\oplus}$ neben den beiden Influenzavirus-A-Stämmen beide kozirkulierenden Influenzavirus-Typ-B-Linien enthält. Damit sind alle Geimpften bestmöglich vor der Grippeinfektion geschützt. Vaxigrip Tetra ${ }^{\circledast}$ ist in 24 Ländern in dieser Grippesaison verfügbar. Die gesamte Impfstoffmenge für die kommende Grippesaison 2017/18 ist bereits vom Paul-EhrlichInstitut freigegeben. Vaxigrip Tetra ${ }^{\circledast}$ ist als Fertigspritze in 1er- und 10er-Packungen lieferbar.

Red.

- Nach Informationen von Sanof

\section{Damit Schokolade essen wieder Spaß macht}

\section{Depressionen überwinden}

Die Therapie von Depressionen hat zum Ziel, negative Emotionen zu beseitigen und die Fähigkeit wiederherzustellen, Freude zu empfinden. Beides zusammen gelingt mit adrenergen und dopaminergen Substanzen besser als mit den häufig verschriebenen selektiven Serotoninwiederaufnahmehemmern (SSRI), erklärte Prof. Göran Hajak, Klinikum Bamberg.

Geht man der Frage nach, welche Erwartungen Patienten an eine Therapie haben, so zeigt sich, dass die Abwesenheit der typischen depressiven Symptome auf Platz drei stehen. Noch wichtiger sind den Patienten die Rückkehr zum gewohnten Funktionsniveau sowie zu einer positiven mentalen Gesundheit mit Optimismus, Kraft und Selbstvertrauen.

Viele Antidepressiva beseitigen effektiv die Niedergeschlagenheit, wirken aber nicht ausreichend gegen die Anhe- donie. Diese Patienten sind dann nicht mehr traurig, aber es fehlt an Freude, Interesse und Tatendrang.

Nicht wenige Antidepressiva verursachen Nebenwirkungen, darunter sexuelle Störungen, QTc-Zeit-Verlängerung und Gewichtszunahme. Agomelatin (Valdo$\mathrm{xan}^{\circledast}$ ) ist laut Hajak ein Antidepressivum, das in der Regel frei von diesen Nebenwirkungen ist und positive Emotionen sowie die soziale Funktionsfähigkeit verbessert. In Studien verminderte es Anhedonie deutlich stärker als Venlafaxin und normalisierte die emotionale Schwingungsfähigkeit signifikant besser als Escitalopram. Hajak veranschaulichte: „Der Patient isst nicht nur wieder Schokolade, sondern sie schmeckt ihm auch, und er fühlt sich gut dabei."

\section{Dr. Dirk Einecke} - Symposium „Management von Depressionen in der Hausarztpraxis", 123. Kongress der Dt. Gesellschaft für Innere Medizin; Mannheim, April 2017 (Veranstalter: Servier) 